\title{
Properties and asymptotic behaviour of the solutions of coupled diffusion equations with time-periodic, space-independent coefficients, with an application to electrodiffusion
}

\author{
By Bengt Nagel
}

\begin{abstract}
CONTE N T S
I. Introduction and statement of problem. Survey of sections II to V $\mathbf{3 6 4}$

II. Existence, uniqueness, and some properties of the Markovian process related to eq. (13) . . . . . . . . . . . . . . 368

III. Properties of $P(t)$, especially the asymptotic behaviour in the timeperiodic case.................... . 376

IV. Asymptotic behaviour of the Markovian process for large $t$ in the time-periodic case... . . . . . . . . . . . . . 383

V. Application to electrodiffusion. Generalizations . . . . . . . . . 388 Acknowledgements . . . . . . . . . . . . . . 390 References... . . . . . . . . . . . . . 390
\end{abstract}

\section{S U M M A R Y}

We study a Markovian process, the state space of which is the product of a set of $n$ points and the real $x$-axis. Under certain regularity conditions this study is equivalent to investigating the solution of a set of coupled diffusion equations, generalization of the Fokker-Planck (or second Kolmogorov) equation. Assuming the process homogeneous in $x$, but in general timeinhomogeneous, this set of equations is studied with the help of the Fourier transformation. The marginal distribution in the $n$ discrete states corresponds to a time-inhomogeneous $n$-state Markov chain in continuous time. The properties of such a Markov chain are studied, especially the asymptotic behaviour in the time-periodic case. We obtain a natural generalization of the well-known asymptotic behaviour in the time-homogeneous case, finding a subdivision of the states into groups of essential states, the distribution inside each group being asymptotically periodic and independent of the starting distribution. Next, still assuming time-periodicity, we study the asymptotic behaviour of the complete Markovian process, showing that inside each of the groups mentioned above the distribution approaches a common normal distribution in $x$-space, with mean value and variance proportional to $t$. Explicit expressions for the proportionality factors are derived.

The general theory is applied to the electrodiffusion equations, corresponding to $n=2$. 


\section{B. NAGEL, Solutions of coupled diffusion equations}

\section{Introduction and statement of problem. Survey of sections II to V}

With "electrodiffusion" in the wide sense one can understand the combined effect of a dynamic chemical equilibrium, a transport process effected e.g. by an external electric field, and ordinary diffusion. One of the interesting features here is that if the different substances in equilibrium have different mobilities in the external field, the effective diffusion, superposed on the transport process, can get a considerable contribution from the combined effect of the chemical transition and the transport mechanisms.

The simplest case, the only one treated so far, is where we have a solution of a substance $A$, which can exist in two ionic forms, $A_{1}$ and $A_{2}$, forming a system in chemical equilibrium $A_{1} \rightleftharpoons A_{2}$, determined by transition constants $k_{1}$ and $k_{2} . A_{1}$ and $A_{2}$ have diffusion constants $D_{1}$ and $D_{2}$ and mobilities $u_{1}$ and $u_{2}$ in an external electric field $g(t)$, which we shall assume to be constant in space but possibly time-dependent.

This process, in one space dimension, has been studied (Bak, Kauman [1] Ljunggren [2]), starting from the intuitively natural combination of chemical equilibrium and diffusion equations

$$
\left.\begin{array}{c}
\frac{\partial c_{1}}{\partial t}=-k_{1} c_{1}+k_{2} c_{2}-u_{1} g(t) \frac{\partial c_{1}}{\partial x}+D_{1} \frac{\partial^{2} c_{1}}{\partial x^{2}} \\
\frac{\partial c_{2}}{\partial t}=k_{1} c_{1}-k_{2} c_{2}-u_{2} g(t) \frac{\partial c_{2}}{\partial x}+D_{2} \frac{\partial^{2} c_{2}}{\partial x^{2}} .
\end{array}\right\}
$$

$c_{1}$ and $c_{2}$ are the concentrations of $A_{1}$ and $A_{2}$.

Assuming $g(t)$ constant or periodic, and also making some further simplifying assumptions, the authors quoted above study the behaviour of $c_{1}$ and $c_{2}$ for large $t$, especially the mean values and variances (giving the effective transport and diffusion rates) of these distributions. Although many interesting results are obtained, the solutions are not entirely complete and general.

The electrodiffusion problem has also been attacked by several authors using more direct statistical methods. Starting from a two-state random walk model and assuming $g(t)$ periodic, Thedéen [3] has given a complete description of the asymptotic behaviour for large $t$, including a proof of the asymptotic normality of the position variable and explicit expressions for the mean value and the variance. Thedéen's results agree with the results obtained from (1), when restricted to the special cases treated with the help of (1).

Considering the well-known close connection between ordinary random walk and the usual diffusion equation, it is natural to expect an analogous connection to hold also in this more general case.

Making an obvious generalization from 2 to $n$, we study in the following a not necessarily time-homogeneous Markovian process, where the state space is the (settheoretical) product of a set of $n$ points and the real axis. Assuming for simplicity that the probability densities exist (this is actually not a restriction, as they always exist as positive measures, and all equations remain true in this case as they stand if they are taken in the distribution [generalized function] sense), the distribution at a time $t$ is given by a column vector $p(x, t)=\left\{p_{1}(x, t), \ldots p_{n}(x, t)\right\}$, where $p_{i}(x, t)$ are non-negative and 


$$
\sum_{i} \int p_{i}(x, t) d x=1
$$

(All integrations are from $-\infty$ to $\infty$, if not otherwise indicated.) Introducing the column vector $a=\{1,1, \cdot 1\}$ and denoting transposition by ${ }^{T}$, we can write the last condition as

$$
a^{T} \int p(x, t) d x=1
$$

The process is governed by the transition function, in our case an $n \times n$ matrix

$$
Q(x, t ; y, \tau)=\left(q_{i k}(x, t ; y, \tau)\right), \text { defined for } t>\tau,
$$

giving the distribution at time $t$ from the distribution at an earlier time $\tau$ through the Chapman-Kolmogorov equation

$$
p(x, t)=\int Q(x, t ; y, \tau) p(y, \tau) d y
$$

As $p(x, t)$ should be a probability density if $p(y, \tau)$ is one, $Q$ must have the properties

$$
q_{i k}(x, t ; y, \tau) \geqslant 0 \quad \text { and } \quad a^{T} \int Q(x, t ; y, \tau) d x=a^{T}
$$

(2) is essentially equivalent to

$$
Q\left(x, t ; z, t_{0}\right)=\int Q(x, t ; y, \tau) Q\left(y, \tau ; z, t_{0}\right) d y, \quad t_{0}<\tau<t .
$$

(2) or (4) expresses the Markovian character of the process: the probability distribution at a time $t$ is completely determined from a knowledge of the distribution at any one earlier time.

If the process is homogeneous in $x$-space (space translation invariant), homogeneous in time, or periodic in time with period $\omega$, we have

$$
\begin{aligned}
Q(x, t ; y, \tau)= & Q_{1}(x-y, t, \tau) \\
& Q_{2}(x, y, t-\tau) \\
& Q(x, t+\omega ; y, \tau+\omega), \quad \text { respectively. }
\end{aligned}
$$

If $Q$ fulfills certain regularity requirements as $t \rightarrow \tau$, one can derive from (2) a differential equation for $p(x, t)$, which is the generalization to $n$ states of the ordinary one-state Fokker-Planck (or second Kolmogorov) equation. We refer e.g. to [4], Ch.X, for the derivation in the case $n=1$. The generalization to $n>1$ is obvious: instead of multiplying with an arbitrary function $R(x)$ we use an arbitrary row vector $R^{T}(x)$, multiplied from the left. We only state the requirements on $Q$, which are used in the derivation of the differential equation. Of these only (R2), describing the transition between the discrete states, is peculiar for the case $n>1$. 
B. NAGEL, Solutions of coupled diffusion equations

For every $\delta>0$ we should have (lim means $\lim _{\Delta \tau \rightarrow+0}$ )

1. Continuity:

$$
\lim \frac{1}{\Delta \tau} \int_{|x-y|>\delta} Q(x, \tau+\Delta \tau ; y, \tau) d x=0 .
$$

2. Existence of a local instantaneous transition probability between the discrete states:

$$
\lim \frac{1}{\Delta \tau}\left[\int_{|x-y|<\delta} Q(x, \tau+\Delta \tau ; y, \tau) d x-E\right]=Q(y, \tau)
$$

Here $E$ is the $n \times n$ unit matrix. From (R 1$),(\mathrm{R} 2)$ and (3) follows

$$
q_{i k}(y, \tau) \geqslant 0, i \neq k \text { and } a^{T} Q(y, \tau)=0 .
$$

(3) and (4) require, loosely speaking, the existence of first and second moments:

3.

$$
\begin{aligned}
& \lim \frac{1}{\Delta \tau} \int_{|x-y|<\delta}(x-y) Q(x, \tau+\Delta \tau ; y, \tau) d x=A(y, \tau), \\
& \lim \frac{1}{\Delta \tau} \int_{|x-y|<\delta}(x-y)^{2} Q(x, \tau+\Delta \tau ; y, \tau) d x=B(y, \tau) .
\end{aligned}
$$

Furthermore the convergence in (R1)-(R4) should be uniform in $y$. This condition is automatically fulfilled if the process is homogeneous in space.

Combining (R3) and (R4) with (R 2), observing that $\delta$ can be chosen arbitrarily small, we find that $A$ and $B$ are diagonal matrices. $B$ is evidently non-negative. We assume $Q, A$ and $B$ continuous in $\tau$, and sufficiently regular in $y$ for the final equation (7) to have a meaning.

Using (R1)-(R4) and generalizing the ordinary derivation in the indicated way, we get the equation

$$
\frac{\partial}{\partial t} p(x, t)=Q(x, t) p(x, t)-\frac{\partial}{\partial x}[A(x, t) p(x, t)]+\frac{1}{2} \frac{\partial^{2}}{\partial x^{2}}[B(x, t) p(x, t)] .
$$

Assuming from now on that the process is homogeneous in $x$, we find from (5a) and (R2)-(R4) that $Q, A$ and $B$ are independent of $y$.

The electrodiffusion equation (1) is evidently the special case of (7) where $n=2$, and $Q$ and $B$ are independent also of $t$.

For simplicity we shall in general study a process starting at $t=0$, which is not an essential specialization. Furthermore, to get rid of the special starting distribution for $t=0$, we introduce the fundamental solution of (7), belonging to $t=0$, i.e. an $n \times n$ matrix $G(x, t)$, satisfying

$$
\frac{\partial}{\partial t} G(x, t)=\left[Q(t)-A(t) \frac{\partial}{\partial x}+\frac{1}{2} B(t) \frac{\partial^{2}}{\partial x^{2}}\right] G(x, t),
$$

$\lim _{t \rightarrow+0} G(x, t)=\delta(x) \cdot E, \quad$ where $\delta(x)$ is the delta function. 
With the help of $G(x, t)$ we can express the solution of (7) with initial values $p(x, 0)$ at $t=0$ as

$$
p(x, t)=\int G(x-y, t) p(y, 0) d y
$$

Comparing (9) and (2) we find

$$
G(x-y, t)=Q(x, t ; y, 0)
$$

(8), (8a) is the fundamental equation. We are interested in the properties of the solution $G(x, t)$, especially in the asymptotic behaviour for large $t$.

We recall the conditions on $Q(t), A(t)$, and $B(t)$ :

They are continuous; $Q(t)$ has non-negative off-diagonal elements, and

$a^{T} Q(t)=0 ; A(t)$ and $B(t)$ are diagonal, and $B(t)$ is non-negative.

As the coefficients in (8) are independent of $x$, the obvious thing is to make a Fourier transformation and go over to the characteristic function

$$
P(t, p)=\int e^{i p x} G(x, t) d x
$$

Then (8), (8 a) go over into

$$
\begin{gathered}
\frac{\partial}{\partial t} P(t, p)=\left[Q(t)+i p A(t)-\frac{1}{2} p^{2} B(t)\right] P(t, p), \\
P(0, p)=E
\end{gathered}
$$

For a process, homogeneous in $x$, the right hand side of (4) is easily seen to be a convolution integral. As convolution in $x$-space corresponds to multiplication in $p$-space, $(4)$ is in this case equivalent to

$$
P\left(t, t_{0}, p\right)=P(t, \tau, p) P\left(\tau, t_{0}, p\right), \quad t_{0}<\tau<t,
$$

where $P(t, \tau, p)$ is the characteristic function corresponding to $Q(x, t ; y, \tau)=Q_{1}(x-y, t, \tau)$ [cf. (5a), (10), and (12)].

Equation (13), being a system of ordinary first order linear differential equations with a simple dependence on the parameter $p$, is in general simpler to study than the coupled second order partial differential equations (8). Of course, as $G(x, t)$ is the quantity of most direct physical interest, we have to translate the properties of $P(t, p)$ into the corresponding properties of $G(x, t)$.

In Sect. II we prove that the solution of (13) really gives a unique Markovian process with the properties $(\mathrm{R} 1)-(\mathrm{R} 4)$. We also derive some properties of $P(t, p)$, useful in the following. In Sect. III we study more in detail the behaviour of $P(t)=$ $P(t, 0)$, giving the time behaviour of the distribution in the $n$ states, integrated over $x$, cf. (12). This is a time-continuous, but in general time-inhomogeneous, $n$-state Markov chain. In particular we study the behaviour for large $t$ when the transition function $Q(t)$ is periodic. It turns out, as a natural generalization of the wellknown case where $Q(t)$ is constant, that we get a certain number of "non-interacting" 


\section{B. NAGEL, Solutions of coupled diffusion equations}

groups of final states. Inside each of these groups the distribution is asymptotically periodic and independent of the distribution for $t=0$. The probability division between the different groups depends on the initial distribution, however.

In IV, using the results obtained in III, we study $P(t, p)$ for large $t$ and small $p$, assuming also $A(t)$ and $B(t)$ periodic. From this study follows that inside each of the above-mentioned groups we approach for large $t$ a common normal distribution in $x$-space with mean value and variance proportional to $t$. Asymptotically the whole group diffuses as a homogeneous substance, and at the same time we have a periodic transition between the states of the group (if $Q(t)$ is constant, this periodic transition is absent, of course). The effective diffusion constant is a sum of two terms, one involving $B(t)$ and $Q(t)$, and the other one, which could be called the electrodiffusion component, depending on $Q(t)$ and $A(t)$. As should be expected, the last term is zero if the section of $A(t)$ which corresponds to the group of states in question has all elements in the diagonal equal.

Finally, in V we apply the formulas obtained in IV to the original electrodiffusion problem (1). The results are in complete agreement with those given by Thedéen [3].

It should be pointed out that this electrodiffusion process is the simplest nontrivial application of the theory studied in this paper (the case $n=1$ being trivial, of course, as we can in this case give an explicit form for the solution of (8), valid for all $t$; cf. [4], Ch.X). As $n=2$ and $Q(t)$ is constant, most of the work in Sect. III concerning the behaviour of $P(t)$ can be bypassed.

At the end of $V$ we indicate some possible generalizations.

\section{Existence, uniqueness, and some properties of the Markovian process related to eq. (13)}

It is known from the theory of linear differential equations that the system of equations (13) with initial conditions (13a) and continuous coefficients has for every $p$ (even complex) a unique solution, with a continuous first derivative in $t$. Moreover, as the coefficients are integral functions of $p$ and the initial conditions are independent of $p, P(t, p)$ is for every $t$ an integral function of $p$. It is also easy to see that every derivative $\left(\partial^{n} P / \partial p^{n}\right)(t, p)$ is continuous and has a continuous first derivative as function of $t$. These functions as well as $P(t, p)$ are simultaneously continuous in $t$ and $p$; hence we may interchange the order of differentiation between $\partial / \partial t$ and $\partial^{n} / \partial p^{n}$.

From the work in I leading to (13) follows that if there exists a Markovian process (i.e. a function or, more precisely, a measure $Q(x, t ; y, \tau)$ satisfying (3) and (4)) with the properties (5a) and (R 1$)-(\mathrm{R} 4)$, where $Q, A$, and $B$ are now assumed independent of $y$, it must be given by the inverse Fourier transform of the unique solution $P(t, \tau, p)$ of (13), fulfilling the condition (13a) at time $t=\tau$ instead of $t=0$. Hence uniqueness is ensured.

The proof of existence is not quite as simple. What we have to prove is that if $Q, A$, and $B$ are functions fulfilling conditions (11), the solution $P(t, \tau, p)$ of (13) really defines a Markovian process with the desired properties, i.e. that the inverse Fourier transform $Q_{1}(x-y, t, \tau)=Q(x, t ; y, \tau)$ exists as a measure satisfying (3), (1), and $(\mathrm{R} 1)-(\mathrm{R} 4)$. The verification of the second part of $(3),(4)$, and $(\mathrm{R} 1)-(\mathrm{R} 4)$ is rather simple. The crucial point is to prove that the matrix elements of $Q_{1}(x-y, t, \tau)$ are (bounded) positive measures. 
According to Bochner's theorem (see. e.g. [4] Ch. VII) this is equivalent to proving that the matrix elements of $P(t, \tau, p)$ are, for $t>\tau$, positive definite functions of $p$. (For shortness we shall in the following often omit the phrase "the matrix elements of" and simply write " $P(t, \tau, p)$ is positive definite", though, strictly speaking, this statement when applied to a matrix may have another meaning; the same remark also applies to the concepts "positive" and "non-negative".)

We recall that a function $f(p)$, defined on the real axis, is called positive definite if it is continuous and if for any set of real points $\left(p_{1}, \ldots p_{m}\right)$ and corresponding arbitrary complex numbers $\left(\xi_{1}, \ldots \xi_{m}\right)$ we always have

$$
\sum_{i, k} f\left(p_{i}-p_{k}\right) \xi_{i} \xi_{k} \geqslant 0
$$

As we shall have no reason to introduce more general positive definite functions than continuous ones, we include for shortness of expression the continuity condition in the definition of positive definiteness.

One of the simplest properties of a positive definite function, which follows directly from (15), is that it is uniformly bounded on the real axis, more precisely

$$
|f(p)| \leqslant f(0), \quad f(0) \text { real and } \geqslant 0 .
$$

The theorem of Bochner states that the Fourier transformation

$$
f(p)=\int e^{i p x} d \mu(x)
$$

establishes a one-to-one correspondence between the set of bounded positive measures on the real axis and the set of positive definite functions.

For simplicity we prove the positive definiteness of $P(t, p)$, solution of (13), (13a), but it is evident that the proof carries over directly to the general case $P(t, \tau, p)$.

In the proof we need some simple properties (see e.g. [5], Ch. XIV) of the solution $R(t)$ of the matrix equation

$$
\frac{d}{d t} R(t)=S(t) R(t), \quad R(0)=E .
$$

Here $S(t)$ is assumed to be continuous and in general complex.

First we state a result which will be used in Sect. III. The determinant $|R(t)|$ is given by the Jacobian identity

$$
|R(t)|=\exp \left[\sum_{1}^{n} \int_{0}^{i} s_{i i}(\tau) d \tau\right]|R(0)|
$$

which shows that $R(t)$ is non-singular. If $S(t)$ has real diagonal elements $|R(t)|$ is real and positive.

(18) is equivalent to the integral equation

$$
R(t)=E+\int_{0}^{t} S(\tau) R(\tau) d \tau,
$$




\section{B. NAGEL, Solutions of coupled diffusion equations}

which can be solved by iteration, and we then obtain the solution in the form of an infinite series

$$
R(t)=E+\int_{0}^{t} d \tau_{1} S\left(\tau_{1}\right)+\int_{0}^{t} d \tau_{1} S\left(\tau_{1}\right) \int_{0}^{\tau_{1}} d \tau_{2} S\left(\tau_{2}\right)+\ldots
$$

which is absolutely convergent for all $t$.

The explicit solution (21) will be used in the proof.

Besides we need some simple facts about positive definite functions:

a. 1 is pos. def.; if $f(p)$ is pos. def., then so is $k \cdot f(p), k \geqslant 0$.

b. A linear combination (a finite sum or, with some obvious precautions, an integral), with non-negative coefficients, of pos. def. functions is again pos. def.

c. If $f(p)$ is pos. def., then so is $e^{i \alpha p} f(p), \alpha$ real.

$(a)$ and $(c)$ imply that $e^{i \alpha p}$ is pos. def.

d. $\exp \left(-\beta p^{2}\right), \beta \geqslant 0$, is pos. def.

e. If $\left\{f_{n}(p)\right\}$ is a sequence of pos. def. functions, converging in every point to a continuous function $f(p)$, then $f(p)$ is pos. def.

$(a),(b),(c)$, and $(e)$ are easily proved directly from the defining condition (15). (c) also follows, with the help of Bochner's theorem, from the fact that a translation a distance $\alpha$ of the measure in $x$-space corresponds to a multiplication with $\exp (i \alpha p)$ in $p$-space. Finally, $(d)$ is a consequence of the fact that $\exp \left(-\beta p^{2}\right), \beta>0$, is (apart from a positive factor) the Fourier transform of $\exp \left(-x^{2} / 4 \beta\right)$.

We can now prove

Theorem 1. If $Q(t), A(t)$, and $B(t)$ satisfy the conditions (11), then the matrix elements of $P(t, p)$, solution of $(13)$ and $(13 a)$, are for every $t \geqslant 0$ positive definite functions of $p$.

Remark. The condition $a^{T} Q(t)=0$ is not used in the proof.

Proof. The continuity requirement is evidently fulfilled.

Before using formula (21) we make a transformation in (13), putting

$$
P(t, p)=\exp \left[-m t E+i p \int_{0}^{t} A(\tau) \mathrm{d} \tau-\frac{1}{2} p^{2} \int_{0}^{t} B(\tau) d \tau\right] P^{\prime}(t, p) .
$$

Here $m$ is chosen so that $m E+Q(t)$ has non-negative diagonal elements in the whole interval $(0, t)$ that we study. One should notice that all three terms in exp are diagonal and hence commuting matrices, so that the usual law $\exp (a+b)=\exp a \cdot \exp b$ is valid. For shortness we use the notation

$$
D(t)=\exp \left[-\frac{1}{2} p^{2} \int_{0}^{t} B(\tau) d \tau\right]
$$

where the diagonal elements of $D(t)$ are

$$
d_{i}(t)=\exp \left[-\frac{1}{2} p^{2} \int_{0}^{t} b_{i}(\tau) d \tau\right]
$$

We shall prove that $D(t) \cdot P^{\prime}(t, p)$ is positive definite. Then properties $(a)$ and $(c)$ above imply that $P(t, p)$ is positive definite. 
The transformation (22) in (13) leads to a similar equation for $P^{\prime}(t, p)$, with the first factor on the right hand side of (13) substituted by

$$
Q^{\prime}(t)=D^{-1}(t) \exp \left[-i p \int_{0}^{t} A(\tau) d \tau\right][Q(t)+m E] \exp \left[i p \int_{0}^{t} A(\tau) d \tau\right] D(t) .
$$

Applying the solution formula (21) and multiplying by $D(t)$ we get

$$
D(t) \cdot P^{\prime}(t, p)=D(t)+\int_{0}^{t} d \tau_{1} D(t) Q^{\prime}\left(\tau_{1}\right)+\int_{0}^{t} d \tau_{1} \int_{0}^{\tau_{2}} d \tau_{2} D(t) Q^{\prime}\left(\tau_{1}\right) \cdot Q^{\prime}\left(\tau_{2}\right)+\ldots
$$

If we study a certain matrix element of a term on the right hand side of (26), we obtain from the repeated matrix multiplication a sum of terms, where each term is a product of the non-negative elements of $Q(t)+m E$, purely imaginary exponentials $\exp [i p \alpha(t)]$ coming from the factors $\exp \left[ \pm i p \int_{0}^{t} A(\tau) d \tau\right]$ in $(25)$, and factors of the form $d_{i}$ and $d_{i}^{-1}$ from $D(t)$ in (25) and (26). From $(a)$ and $(c)$ follows that it is the last type of factors which is of importance. A moment's reflection shows that they contribute to each term of a matrix element $\alpha \beta$ a factor of the form

$$
d_{\alpha}(t) d_{\alpha}^{-1}\left(\tau_{1}\right) d_{i_{1}}\left(\tau_{1}\right) d_{i_{1}}^{-1}\left(\tau_{2}\right) \ldots d_{\beta}\left(\tau_{\nu}\right) .
$$

Except for the last factor $d_{\beta}$, the factors in (27) can be paired together in the form

$$
d_{i}(\tau) d_{i}^{-1}\left(\tau^{\prime}\right), \quad \tau \geqslant \tau^{\prime} .
$$

From (24) and the non-negativity of $b_{i}(\tau)$ follows that every such pair, and hence the whole product $(27)$, is of the form $\exp \left(-p^{2} \beta\right)$, where $\beta$ is a function of $t, \tau_{1}, \tau_{2}, \ldots$, non-negative in the integration region. In every term in (26) the integrand is thus positive definite, and as the integration evidently gives a continuous function of $p,(b)$ shows that every term on the right hand side of (26) is positive definite, thus also the partial sums, and from the convergence for every $p$ of the series (26) and property $(e)$ we conclude that $D(t) \cdot P^{\prime}(t, p)$ is positive definite, and then so is $P(t, p)$. The proof is complete.

It could be remarked that although $P(t, p)$ is of course uniquely defined and an integral function of $p$ also for $t<0$, it is then in general no longer positive definite.

Generalizing to $P(t, \tau, p)$ and applying Bochner's theorem we find that $P(t, \tau, p)$ is, for every $t \geqslant \tau$, the Fourier transform of a bounded positive measure $Q_{1}(x-y, t, \tau)$. Hence the first property in (3) is established. (4) is equivalent to (14), which is a trivial consequence of (13). (14) only means that we can perform the integration of (13) from $t_{0}$ to $t$ in two steps, going first from $t_{0}$ to $\tau$ and then from $\tau$ to $t$. There only remains to prove the second relation in $(3)$ and $(\mathbf{R} 1)-(\mathbf{R} 4)$. Again we put $\tau=0$ without losing generality.

(12) shows that the second condition (3), expressed in $P(t, p)$, takes the form

$$
a^{T} P(t, 0)=a^{T} .
$$

But (29) follows directly from (13) and $a^{T} Q(t)=0$.

As $P(t, p)$ is analytic for all $p$, it is infinitely differentiable for $p=0$, and (12) then shows that all moments of $G(x, t)$ exist for $t \geqslant 0$ and are given by 
B. NAGEL, Solutions of coupled diffusion equations

$$
\int x^{n} G(x, t) d x=i^{-n}\left(\partial^{n} P / \partial p^{n}\right)(t, 0), \quad n=0,1,2, \ldots
$$

(13a) gives

$$
P(0,0)=E ;\left(\partial^{n} P / \partial p^{n}\right)(0,0)=0, \quad n=1,2, \ldots
$$

(30), (31) and the remark at the beginning of this section about the existence of the first time derivative of $\partial^{n} P / \partial p^{n}$ shows that

$$
\lim _{\Delta \tau \rightarrow 0} \frac{1}{\Delta \tau}\left[\int x^{n} G(x, \Delta \tau) d x-\delta_{o n} E\right]=i^{-n}\left(\partial^{n+1} P / \partial t \partial p^{n}\right)(0,0) .
$$

( $\delta_{o n}$ is Kronecker's delta).

The right hand side of (32) is easily evaluated with the help of (13), remembering that we may interchange $\partial / \partial t$ and $\partial^{n} / \partial p^{n}$. In this way one easily establishes $(\mathbf{R} 2)-$ (R4), except for the fact that one should integrate over $|x|<\delta$ only and not over all $x$, as indicated on the left hand side of (32). However, applying (32) to the case $n=4$, we find

$$
\lim \frac{1}{\Delta \tau} \int x^{4} G(x, \Delta \tau) d x=0
$$

and this implies

$$
\lim \frac{1}{\Delta \tau} \int_{|x|>\delta} x^{y} G(x, \Delta \tau) d x=0, \quad \nu=0,1,2,3,4
$$

for every $\delta>0$.

$v=0$ gives $(\mathrm{Rl})$, and $v=0,1$, and 2 show that the extension of the integrals in (R2)-(R4) to the whole axis does not change the values.

Altogether, we have proved the following theorem:

Theorem 2. (Existence and uniqueness.)

For every set $Q(t), A(t)$, and $B(t)$ satisfying conditions (11), there exists one and only one Markovian process (i.e. a measure-valued transition function $Q(x, t ; y, \tau)=$ $Q_{1}(x-y, t, \tau), t>\tau$, satisfying (3) and (4)) fulfilling $(R 1)-(R 4)$ with the given $Q, A$, and $B . Q_{1}(x-y, t, \tau)$ has the characteristic function $P(t, \tau, p)$, defined as the solution of (13) with initial value $P(\tau, \tau, p)=E$.

We shall now study some further properties of $G(x, t)$, related to the behaviour for $x \rightarrow \pm \infty$ and to the local regularity.

(30) shows that for every $t \geqslant 0$ moments of all orders exist. As $P(t, p)$ is an integral function, it is well-known that the Fourier transformation formula (12) can be extended to arbitrary complex values of $p$. In fact, as the series expansion around $p=0$ of $P(t, p)$ is absolutely convergent for all $p$, this implies the convergence of

$$
\sum_{0}^{\infty} \frac{m_{n}}{n} R^{n}, \quad \text { for any positive } R, \text { arbitrarily large. }
$$

Here $m_{n}$ represents the absolute value of some matrix element of the moment of order $n$. It is then easy to see that the right hand side of (12) converges and defines 
an integral function (which must be equal to $P$ ) even if we use complex values, say $p+i q, p$ and $q$ real. If we put $p=0, P(t, i q)$ then being real and positive, we find

$$
\int e^{-q x} G(x, t) d x<\infty, \text { all real } q,
$$

i.e. $G(x, t)$ vanishes faster than any exponential as $x \rightarrow \pm \infty$.

Later on we shall sharpen (36), showing that $G(x, t)$ in fact vanishes faster than some Gaussian $\exp \left(-b x^{2}\right)$.

If the initial distribution $p(y, 0)$ has finite moments including order $m$, say, relation (9), or better the Fourier transformed relation ( denotes Fourier transformation)

$$
\tilde{p}(t, p)=P(t, p) \tilde{p}(0, p)
$$

shows that $p(x, t)$ has also finite moments including order $m$. Namely, if $\tilde{p}(0, p)$ has derivatives in $p$ at $p=0$ including order $m$, then so has $\tilde{p}(t, p)$, as $P(t, p)$ is analytic.

We have thus found that as a consequence of $P(t, p)$ being, as function of $p$, very regular locally, $G(x, t)$ is well-behaved (decreases rapidly) at infinity.

Conversely, the behaviour of $P(t, p)$ as $p \rightarrow \pm \infty$ is in a similar way related to the local regularity of $G(x, t)$.

Without any further conditions on $Q, A$, and $B$ we can say nothing more than that $G(x, t)$ is a positive measure, as is shown by the trivial case $B(t)=0, A(t)=a(t) E$, which has the solution

or

$$
\begin{gathered}
P(t, p)=\exp \left[i p \int_{0}^{t} a(\tau) d \tau\right] P(t), \quad P(t)=P(t, 0) \\
G(x, t)=\delta\left(x-\int_{0}^{t} a(\tau) d \tau\right) P(t) .
\end{gathered}
$$

However, if we add the assumption, reasonable in physical applications, that the diagonal elements of $B(t)$ are positive (or positive simultaneously at least at one time), it is not difficult to show that $G(x, t)$ is very regular, indeed an integral function of $x$.

To show this we can use the following simple comparison theorem for systems of linear differential equations:

Theorem 3. Given a continuous real matrix $S(t)$ with non-negative elements outside the diagonal. Then $R(t)$, solution of

$$
\frac{d}{d t} R(t)=S(t) R(t), \quad R(0)=E
$$

is for $t \geqslant 0$ non-negative and has positive diagonal elements.

If $S^{\prime}(t)$ is another continuous matrix, which may be complex, and if

$$
\operatorname{Re} s_{i i}^{\prime}(t) \leqslant s_{i i}(t), \quad\left|s_{i k}^{\prime}(t)\right| \leqslant s_{i k}(t), \quad i \neq k,
$$

then for $R^{\prime}(t)$, solution of 
B. NAGEL, Solutions of coupled diffusion equations

$$
\frac{d}{d t} R^{\prime}(t)=S^{\prime}(t) R^{\prime}(t), \quad R^{\prime}(0)=I,
$$

we have

$$
\left|r_{i k}^{\prime}(t)\right| \leqslant r_{i k}(t) \text { for } t \geqslant 0 \text {. }
$$

Proof. Put

$$
R(t)=e^{-m t} R_{1}(t)
$$

in (39), where $m$ is chosen so that $S(t)+m E$ is non-negative. Applying (21) to $R_{1}(t)$ we find that all terms on the right hand side of (21) are non-negative, while the first term $E$ ensures positivity of the diagonal elements. The statement of therfirst part of the theorem is then true for $R_{1}(t)$, and thus also for $R(t)$.

For the second part of the theorem we may imagine that we have already performed a transformation of type (43), with the same $m$ in both cases, on $R(t)$ and $R^{\prime}(t)$, so that $R e s_{i i}^{\prime}(t) \geqslant 0$. This transformation evidently leaves invariant the relation (42) which is to be proved. Next, we transform away the imaginary parts of the diagonal elements of $S^{\prime}(t)$ by putting

$$
R^{\prime}(t)=D(t) R^{\prime \prime}(t)
$$

where $D(t)$ is diagonal with elements

$$
d_{i}(t)=\exp \left[i \int_{0}^{t} \operatorname{Im} s_{i i}^{\prime}(\tau) d \tau\right] .
$$

As $\left|d_{i}(t)\right|=1$, we have $\left|r_{i k}^{\prime \prime}(t)\right|=\left|r_{i k}^{\prime}(t)\right|$ and for $R^{\prime \prime}(t)$ we get

$$
\frac{d}{d t} R^{\prime \prime}(t)=S^{\prime \prime}(t) R^{\prime \prime}(t), \quad R^{\prime \prime}(0)=E,
$$

where $S^{\prime \prime}(t)$ has real diagonal elements fulfilling

and

$$
\begin{gathered}
0 \leqslant s_{i i}^{\prime \prime}(t) \leqslant s_{i i}(t), \\
\left|s_{i k}^{\prime \prime}(t)\right| \leqslant s_{i k}(t), \quad i \neq k .
\end{gathered}
$$

Expressing the solution of (46) with the help of the series (21), it is easily realized, considering (47) and (48), that we get upper bounds of the absolute values of the matrix elements of $R^{\prime \prime}(t)$ by substituting $s_{i k}(t)$ for $s_{i k}^{\prime \prime}(t)$ everywhere in the series. This proves the second part of the theorem.

Applying the first part of Theorem 3 to $P(t)=P(t, 0)$, solution of

$$
\frac{d}{d t} P(t)=Q(t) P(t), \quad P(0)=E,
$$

we find that $P(t)$ is non-negative (this is also a consequence of Theorem 1 , ef. (16)) and has positive diagonal elements. Furthermore we found in (29) that

$$
a^{T} P(t)=a^{T} \text {. }
$$

A non-negative matrix having the property (50), i.e. such that the sum of the elements in a column is 1 for every column, is called a stochastic matrix, as it can 
be used as transition matrix of an $n$-state Markov chain in discrete time. $P(t)$ is thus, for every $t \geqslant 0$, a stochastic matrix with positive diagonal elements and positive determinant, cf. (19). We shall return to a study of $P(t)$ in Section III.

An application of the second part of Theorem 3 gives

Theorem 4. If $\alpha(t)=\max \left\{\left|a_{1}(t)\right|, \ldots\left|a_{n}(t)\right|\right\}, b(t)=\min \left\{b_{1}(t), \ldots b_{n}(t)\right\}$, and $\beta(t)=$ $\max \left\{b_{1}(t), \ldots b_{n}(t)\right\}$, where $\left\{a_{i}(t)\right\}$ and $\left\{b_{i}(t)\right\}$ are the diagonal elements of $A(t)$ and $B(t)$, respectively, we have the following majorization of the elements of $P(t, p+i q)(p, q$ real $)$ :

$$
\left|p_{i k}(t, p+i q)\right| \leqslant p_{i k}(t) \exp \left[-\frac{1}{2} p^{2} \int_{0}^{t} b(\tau) d \tau+\frac{1}{2} q^{2} \int_{0}^{t} \beta(\tau) d \tau+|q| \int_{0}^{t} \alpha(\tau) d \tau\right] .
$$

Proof. Substituting $p+i q$ for $p$ in the first factor on the right hand side of (13) we get the expression

$$
Q(t)-q A(t)-\frac{1}{2} p^{2} B(t)+\frac{1}{2} q^{2} B(t)+i[p A(t)-p q B(t)] .
$$

The substitution

$$
P(t, p+i q)=\exp \left[-\frac{1}{2} p^{2} \int_{0}^{t} b(\tau) d \tau+\frac{1}{2} q^{2} \int_{0}^{t} \beta(\tau) d \tau+|q| \int_{0}^{t} \alpha(\tau) d \tau\right] P^{\prime}(t, p+i q)
$$

then leads to an equation for $P^{\prime}$, which together with (49) forms a set of equations fulfilling the conditions of Theorem 3. Thus we have $\left|p_{i k}^{\prime}(t, p+i q)\right| \leqslant p_{i k}(t)$, and the theorem is proved.

If we put $q=0$ in (51) and assume that

$$
b=\frac{1}{2} \int_{0}^{t} b(\tau) d \tau
$$

is positive, i.e. that at some point in $(0, t)$ all $b_{i}(\tau)$ are positive simultaneously, we see that $P(t, p)$ decreases faster than some Gaussian function as $p \rightarrow \pm \infty$. Taking the inverse of $(12)$ we then find that $G(x, t)$ is very regular, in fact one can extend the inverse Fourier transform to all complex values $z=x+i y$, and $G(z, t)$ is then an integral function.

In the same way as for $G(x, t)$ it follows from (37) that for any initial distribution $p(x, 0), p(x, t)$ is an integral function of $x$ if $b>0 . \tilde{p}(0, p)$ is positive definite and hence bounded, cf. (16).

So far we have used the inequality (51) only for $q=0$. If we exploit (5l) also for $q \neq 0$, we obtain an improved estimate of the decrease of $G(x, t)$ as $x \rightarrow \pm \infty$. (51) evidently implies that for any number

$$
c>\frac{1}{2} \int_{0}^{t} \beta(\tau) d t \geqslant 0
$$

we can find a number $M_{i k}$ (depending, in general, on $c$ ) so that

$b$ is given by (54).

$$
\left|p_{i k}(t, p+i q)\right| \leqslant M_{i k} e^{-b p^{2}+c q^{2}} .
$$

Let us first assume $b>0$. Then we have seen that $G(z, t)$ is an integral function, and (56) implies the inequality

$$
\left|g_{i k}(x+i y, t)\right| \leqslant M_{i k}(4 \pi b)^{-\frac{1}{2}} \exp \left(-x^{2} / 4 c+y^{2} / 4 b\right) .
$$




\section{B. NAGEL, Solutions of coupled diffusion equations}

(57) follows from (56) and the Fourier integral expressing $g_{i k}$ in $p_{i k}$, if we integrate along the line $p-i x / 2 c,-\infty<p<\infty$.

In the case $b=0$, where $G(x, t)$ may be just a positive measure, we have the weaker result

$$
\begin{gathered}
\int \exp \left(x^{2} / 4 c\right) G(x, t) d x<\infty \\
c>\frac{1}{2} \int_{0}^{t} \beta(t) d \tau .
\end{gathered}
$$

for every

(58) is an immediate consequence of the following simple theorem.

Theorem 5. If $f(p)$ is a positive definite integral function, fulfilling on the imaginary axis

$$
f(i q)<A e^{b^{\prime} q^{2}}, \text { for some } A>0 \text { and } b^{\prime} \geqslant 0,
$$

then for the positive measure $\mu(x)$ associated with $f(p)$ by Bochner's theorem we have

$$
\int \exp \left(x^{2} / 4 b\right) d \mu(x)<\infty \quad \text { for every } \quad b>b^{\prime} .
$$

Proof. Follows from

$$
\int \exp \left(-b q^{2}\right) \cdot f(i q) d q<\infty
$$

if we introduce $f(i q)$ expressed in $\mu(x)$ and interchange the order of integration, which is easily seen to be allowed.

We have shown that $b=\frac{1}{2} \int_{0}^{t} b(\tau) d \tau>0$ is a sufficient condition for $G(x, t)$ to be an integral function. It is probable that this property of $G(x, t)$ holds also under weaker conditions on $B(t)$; in this case one must instead impose some extra condition on $Q(t)$. The following conjecture seems reasonable:

If for some $t$ at least one $\int_{0}^{t} b_{i}(\tau) d \tau$ and all elements of $Q(t)$ are different from zero, then $G\left(x, t^{\prime}\right)$ is an integral function of $x$ for $t^{\prime} \geqslant t$.

Added in proof: Dr E. Asplund has kindly pointed out to the author that if we assume $B(t) \equiv 0$, the majorization (51) implies that the support of $G(x, t)$ on the $x$-axis is compact, as follows directly from the Paley-Wiener theorem (in the generalized form valid for distributions; see L. Schwartz, Théorie des distributions, Tome II). More precisely, one can show that $G(x, t)$ is zero outside the interval with endpoints

$$
\int_{0}^{t} \min _{i} a_{i}(\tau) d \tau \text { and } \int_{0}^{t} \max _{i} a_{i}(\tau) d \tau
$$

a result which is physically aimost self-evident.

\section{Properties of $P(t)$, especially the asymptotic behaviour in the time-periodic case}

In this section we shall study the matrix $P(t)=P(t, 0)$, solution of

$$
\frac{d}{d t} P(t)=Q(t) P(t), \quad P(0)=E,
$$


which gives the probability distribution between the $n$ discrete states of the Markovian process at time $t$. As we saw in Sect. II (cf. (50)), $P(t)$ is a stochastic matrix with positive diagonal elements and a positive determinant.

Concerning the properties of stochastic matrices see e.g. [5], Ch. XIII; cf. also [6]. The terminology in this field is not quite uniform; we shall use a mixture of the terminologies of the books mentioned above. Observe that what we call a stochastic matrix is the transposed of a stochastic matrix as defined in [5].

A stochastic matrix $P$ always has one eigenvalue equal to 1 ; this is a direct consequence of

$$
a^{T} P=a^{T}
$$

Furthermore all eigenvalues $\lambda$ satisfy $|\lambda| \leqslant 1$; especially if $P$ has positive diagonal elements, $\lambda=1$ is the only eigenvalue (possibly multiple) with $|\lambda|=1$. These two properties follow easily from the following simple result, which is sometimes called the theorem of Fréchet:

If $p_{\alpha \alpha}$ is the smallest of the diagonal elements of a stochastic matrix $P$, then all eigenvalues of $P$ satisfy

$$
\left|\lambda-p_{\alpha \alpha}\right| \leqslant 1-p_{\alpha \alpha}
$$

As the proof is short we repeat it here:

Assume $\lambda$ an eigenvalue, $\left(x_{1}, \ldots x_{n}\right)$ the corresponding eigenvector from the left, i.e.

$$
\sum_{i} x_{i} p_{i k}=\lambda x_{k}, \quad k=1, \ldots n .
$$

Assume $\left|x_{m}\right|=\max \left\{\left|x_{1}\right|, \ldots\left|x_{n}\right|\right\}$; evidently $\left|x_{m}\right|>0$. Putting $k=m$, we find

$$
\left|\lambda-p_{m m}\right|\left|x_{m}\right|=\left|\sum_{i \neq m} x_{i} p_{i m}\right| \leqslant\left|x_{m}\right| \sum_{i \neq m} p_{i m}=\left|x_{m}\right|\left(1-p_{m m}\right),
$$

or

$$
\left|\lambda-p_{m m}\right| \leqslant 1-p_{m m}
$$

But it is easy to see that if $\lambda$ satisfies this inequality, $\lambda$ also satisfies (64): the circle in the complex $\lambda$-plane defined by (64) contains all the circles obtained in the same way by using the other diagonal elements instead of the smallest one.

It is obvious that a slight modification of the proof gives the result that if $P^{\prime}$ is an arbitrary (complex) matrix, such that the sums of the absolute values of the elements in every column is bounded above by 1 , then every eigenvalue of $P^{\prime}$ satisfies $|\lambda| \leqslant 1$. Using $(51)$ this shows that all eigenvalues of $P(t, p)(p$ real $)$ fulfil $|\lambda| \leqslant 1$.

Our $P(t)$ thus has $\lambda=1$ as only eigenvalue with modulus one. If this is true for a stochastic matrix $P$, the Markov chain determined by $P^{n}, n=1,2$, is called weakly regular (we shall also apply this term to the corresponding $P$ ). In this case

$$
P^{\infty}=\lim _{n \rightarrow \infty} P^{n}
$$

exists, i.e. the Markov chain converges towards an asymptotic distribution. This distribution $p_{\infty}$, determined from the starting distribution $p_{0}$ by

$$
p_{\infty}=P^{\infty} p_{0}
$$




\section{B. NAGEL, Solutions of coupled diffusion equations}

in general depends on $p_{0} . p_{\infty}$ is independent of $p_{0}$ if and only if $\lambda=1$ is a simple eigenvalue. This means that $P^{\infty}$ has all columns $=p_{\infty}$, which is also the unique right eigenvector belonging to the eigenvalue $\lambda=1$ of $P$. We are then in the regular case. A special case here is the positively regular chain, where $p_{\infty}$ has all elements positive.

Given a weakly regular stochastic matrix $P$, with multiplicity $g(\geqslant 1)$ of the eigenvalue 1 , we can by a simultaneous permutation of row and column indices (corresponding to a renumbering of the states of the Markov chain) put it in the following normal form

$$
P=\left(\begin{array}{cc|c}
P_{1} & 0 & P_{1 R} \\
\cdot & \cdot \\
0 & P_{g} & P_{g R} \\
\hline 0 & P_{R}
\end{array}\right)
$$

Here $P_{1}, \ldots P_{g}$ are positively regular stochastic matrices, corresponding to the $g$ groups of essential states, whereas $P_{R}$ is not stochastic. As $P_{1} \ldots P_{g}$ exhaust all eigenvalues 1 of $P$, and $P$ is weakly regular, $P_{R}$ has all eigenvalues smaller than one in modulus, and hence

$$
\lim _{n \rightarrow \infty} P_{R}^{n}=0 .
$$

The states corresponding to $P_{R}$ will be called unessential.

As we know that $\lim P^{n}$ exists, the relation $\lim P^{n+1}=\left(\lim P^{n}\right) P$ shows that

$$
P^{\infty}=\left(\begin{array}{cc|c}
P_{1}^{\infty} & 0 & P_{1}^{\infty} P_{1 R}\left(E-P_{R}\right)^{-1} \\
\cdot & & P_{g}^{\infty} P_{g R}\left(E-P_{R}\right)^{-1} \\
0 & P_{g}^{\infty} & 0
\end{array}\right) .
$$

The criterion for a stochastic matrix $P$ to be positively regular is that for some $m$, $P^{m}$ is positive; then also all $P^{m^{\prime}}, m^{\prime}>m$, are positive. If, as in our case, $P$ has positive diagonal elements, one can always take $m=n-1$ ( $P$ is an $n \times n$ matrix), as follows from the following property:

If $A$ is a non-negative $n \times n$ matrix with positive diagonal elements, then $A^{m}, m \geqslant n$, has zero elements in exactly the same places as $A^{n-1}$.

Proof. Evidently $\left(A^{v}\right)_{i k}>0$ implies $\left(A^{v+1}\right)_{i k}>0$ etc., so that $A^{m}$ has not more zeros than $A^{n-1}$.

Assume now $\left(A^{m}\right)_{i k}>0, i \neq k$, some $m \geqslant n$. This means that at least one term $a_{i i_{1}} a_{i_{1} i_{2}} \ldots$ $a_{i_{m-1}}>0$. It is now evident that if two of the indices in the chain $i, i_{1}, \ldots k$ are equal (as they must be if $m \geqslant n$ ) we can short-cut the elements between these indices (divide them away) and still have a positive term of a matrix product $A^{m^{\prime}}, m^{\prime}<m$. In this way we can go on till there are at most $n-1$ factors left, thus proving that $\left(A^{\nu}\right)_{i k}>0$ for some $\nu \leqslant n-1$. From the first statement in the proof we conclude that $\left(A^{n-1}\right)_{i k}>0$.

We shall now return to our study of $P(t)$. To obtain the structure of the normal form (67) of $P(t)$ we need not solve the equation (62) for $P(t)$ explicitly. It is sufficient to study the corresponding normal form of 


$$
Q_{t}=\int_{0}^{t} Q(\tau) d \tau=\left(\begin{array}{cc|c}
Q_{1} & 0 & Q_{1, g+1} \ldots Q_{1 s} \\
\cdot & & \\
0 & Q_{g} & \ldots \\
\hline 0 & Q_{g+1} \ldots & \\
\hline 0 & 0
\end{array}\right) .
$$

Here $Q_{1} \ldots Q_{s}$ are square matrices such that $\left(Q_{i}+m_{i} E\right)^{n_{i}-1}$ have all elements positive; $m_{i}>0$ is chosen so that the matrix $Q_{i}+m_{i} E$, where $E$ is the unit matrix of the right dimension $\left(n_{i} \times n_{i}\right)$, has positive diagonal elements. This condition ensures that the reduction in the normal form in (69) is carried as far as possible. $Q_{1} \ldots Q_{g}$ have every "column sum" (sum of all elements in a column) equal to zero, whereas in every one of $Q_{g+1} \ldots Q_{s}$ at least one column sum is negative; this means that for every $\nu, g+1 \leqslant \nu \leqslant s$, at least one of the matrices $Q_{1 v} \ldots Q_{v-1, v}$ standing above $Q_{v}$ is non-zero. To perform the reduction to normal form in an actual case, with not too large $n$, it might be useful to form $\left(Q_{t}+m E\right)^{n-1}$, which, if we chose $m$ properly, has no redundant zeros.

We now assert that $P(t)$ has the same normal form as $Q_{t}$, with $P_{R}(t)$ corresponding to $Q_{g+1} \ldots Q_{s}$.

The proof follows rather directly from the series solution (21). We first make a transformation $P(\tau)=e^{-m \tau} P^{\prime}(\tau)$, where $m$ is chosen so that $Q^{\prime}(\tau)=Q(\tau)+m E$ has positive diagonal elements in $(0, t)$. This transformation evidently does not change the normal forms (67) or (69). Applying (21) to the equation determining $P^{\prime}(t)$, and observing that for $0 \leqslant \tau \leqslant t Q^{\prime}(\tau)$ has at least as many zero elements as $Q_{t}^{\prime}$, one realizes that $P^{\prime}(t)$ has no more zero elements than $Q_{t}^{\prime}$, but is certainly zero in the places denoted by 0 in (69). This proves the assertion.

A consequence of this relation between the normal forms of $P(t)$ and $Q_{t}$ is that if $0<\tau<t$, then the normal form of $P(\tau)$ is the same as or a refinement of the normal form of $P(t)$ : a group of essential states of $P(t)$ may split into several groups in $P(\tau)$, or some of the unessential states in $P(t)$ may form a group of essential states in $P(\tau)$. It is also seen that if $Q(t)$ is periodic with period 1, say, then $P(t)$ has for $t>1$ the same normal form as $P(I)$.

We now assume $Q(t)$ periodic, $Q(t+1)=Q(t)$. The normal form of

$$
Q_{t=1}=\int_{0}^{1} Q(\tau) d \tau
$$

determines the normal form of $P(t)$ for $t \geqslant 1$. The equation $(62)$ then decomposes into

$$
\begin{aligned}
& \frac{d}{d t} P_{i}(t)=Q_{i}(t) P_{i}(t), \quad i=1 \ldots g ; \quad P_{i}(0)=E . \\
& \frac{d}{d t} P_{R}(t)=Q_{R}(t) P_{R}(t) ; \quad P_{R}(0)=E, \\
& \frac{d}{d t} P_{i R}(t)=Q_{i}(t) P_{i R}(t)+Q_{i R}(t) P_{R}(t), \quad i=1 \ldots g ; \quad P_{i R}(0)=0 .
\end{aligned}
$$




\section{B. NAGEL, Solutions of coupled diffusion equations}

We first study equations (70) and (71). $P_{i}(t)$ are for $t \geqslant 1$ positively regular, which implies that one is a simple eigenvalue, and all other eigenvalues are smaller than one in modulus. $P_{R}(t)$ has for $t \geqslant 1$ all eigenvalues smaller than one in modulus.

As $Q(t)$ is periodic it is known (see e.g. [7]) that the solutions $P(t)$ have the form

$$
P_{i}(t)=S_{i}(t) \exp R_{i} t, \quad P_{R}(t)=S_{R}(t) \exp R_{R} t,
$$

where $S_{i}(t)$ and $S_{R}(t)$ are periodic, period 1 , and equal to $E$ for $t=0 . S$ and $R$ are not uniquely determined: we have, putting $t=1$, $\exp R=P(1)$, which means that $R$ is the logarithm of $P(1)$ (the logarithm of a matrix can be defined for a non-singular matrix), which is not uniquely defined, due to the many-valuedness of the logarithm. In particular the eigenvalues of $R$ are the logarithms of the eigenvalues of $P(1)$, and hence determined up to multiples of $2 \pi i$.

For the general definition of the function of a matrix, applicable also in cases where the series expansion definition fails, see for instance [5], Ch. V. We shall here write down only the fundamental formula expressing the function $f(A)$ of a matrix $A$ in terms of the scalar function $f(\lambda)$ on the spectrum of $A$ and certain fixed polynomials of $A, Z_{k l}$, which are independent of the function $f$ :

$$
f(A)=\sum_{k=1}^{s}\left[f\left(\lambda_{k}\right) Z_{k 1}+f^{\prime}\left(\lambda_{k}\right) Z_{k 2}+\ldots+f^{\left(m_{k}-1\right)}\left(\lambda_{k}\right) Z_{k, m_{k}}\right] .
$$

$\lambda_{1} \ldots \lambda_{s}$ are the eigenvalues of $A$, with multiplicities $m_{i}$ in the minimal equation of $A$; thus $m_{i}$ is not larger than the ordinary multiplicity of the eigenvalue $\lambda_{i}$.

From the connection between the eigenvalues of $R$ and $P(1)$ follows that every $R_{i}$ has a simple eigenvalue 0 (we choose the principal value of the logarithm), and all other eigenvalues have negative real parts. In the same way all eigenvalues of $R_{R}$ have negative real parts. (74) then shows that we can write for large $t$

$$
\exp R_{i} t=P_{i}^{\infty}+0\left(e^{-\alpha t}\right), \quad \text { some } \quad \alpha>0,
$$

and

$$
\exp R_{R} t=0\left(e^{-\beta t}\right), \quad \text { some } \quad \beta>0 \text {. }
$$

Evidently

$$
P_{i}^{\infty}=\lim _{n \rightarrow \infty}\left[P_{i}(1)\right]^{n} \text {. }
$$

As $P_{i}(1)$ is positively regular, $P_{i}^{\infty}$ has all colums equal and positive, e.g. can be written in the form

$$
P_{i}^{\infty}=p_{i \infty} a^{T}, p_{i \infty} \text { positive. }
$$

(73), (75), and (78) give

$$
P_{i}(t)=p_{i \infty}(t) a^{T}+0\left(e^{-\alpha t}\right)
$$

where we have put

$$
p_{i \infty}(t)=S_{i}(t) p_{i \infty}
$$

As

$$
P_{i}(N+\tau)=P_{i}(\tau)\left[P_{i}(1)\right]^{N},
$$

and hence

$$
p_{i \infty}(\tau) a^{T}=\lim _{N \rightarrow \infty} P_{i}(N+\tau)=P_{i}(\tau) P_{i}^{\infty}
$$

the periodic distribution $p_{i \infty}(t)$ is positive for all $t$. 
In a similar way one finds

$$
\exp \left(R_{i} t\right) p_{i \infty}=p_{i \infty}, \quad \text { all } t
$$

It should be pointed out that except in the case when $Q(t)$ is constant, exp $R t$ is in general not a stochastic matrix for $t \neq 0,1,2 \ldots$. We shall add some remarks related to this question at the end of the section.

(76) shows that the probability of an unessential state goes to zero for large $t$. Asymptotically only the essential states are populated, and inside each of these groups we approach a positive periodic distribution $p_{i \infty}(t)$, which is independent of the distribution for $t=0$. However, the distribution between the $g$ groups of essential states depends on the initial distribution. To see how the initial probability distribu. tion of the unessential states is divided between the groups of essential states we have to solve eq. (72).

Substituting we find from (72)

$$
P_{i R}(t)=P_{i}(t) C_{i R}(t)
$$

$$
\frac{d}{d t} C_{i R}(t)=P_{i}^{-1}(t) Q_{i R}(t) P_{R}(t) ; \quad C_{i R}(0)=0,
$$

or

$$
P_{i R}(t)=P_{i}(t) \int_{0}^{t} P_{i}^{-1}(\tau) Q_{i R}(\tau) P_{R}(\tau) d \tau
$$

Introducing (73) and (75) we can write (86) in the form

$$
\begin{aligned}
P_{i R}(t)= & S_{i}(t) P_{i}^{\infty} \int_{0}^{t} S_{i}^{-1}(\tau) Q_{i R}(\tau) P_{R}(\tau) d \tau \\
& +S_{i}(t) \int_{0}^{t}\left[\exp R_{i}(t-\tau)-P_{i}^{\infty}\right] S_{i}^{-1}(\tau) Q_{i R}(\tau) P_{R}(\tau) d \tau .
\end{aligned}
$$

As $P_{R}(\tau)$ and $\exp R_{i}(t-\tau)-P_{i}^{\infty}$ decrease exponentially for large $\tau$ and $t-\tau$, respectively, whereas the other factors in the integrals are periodic and hence bounded, one finds that the second integral in (87) tends exponentially to zero for large $t$, and the first one approaches a constant value $\bar{P}_{i R}^{\infty}$. This gives, with (78) and (80),

$$
P_{i R}(t)=p_{i \infty}(t) a^{T} \bar{P}_{i R}^{\infty}+0\left(e^{-\gamma t}\right), \text { some } \gamma>0 .
$$

We put

$$
b_{i}^{T}=a^{T} \bar{P}_{i R}^{\infty} .
$$

$b_{i}$ is then a column vector with $n_{R}$ elements $\left(n_{R}\right.$ is the number of unessential states), which are non-negative: as a submatrix of the stochastic matrix $P(t), P_{i R}(t)$ must be non-negative for all $t$.

Collecting the results (79), (88) and (89) we have shown that $P(t)$ goes exponentially towards the asymptotic form

$$
P^{\infty}(t)=\left(\begin{array}{cr|c}
p_{1 \infty}(t) a^{T} & 0 & p_{1 \infty}(t) b_{1}^{T} \\
0 & p_{g \infty}(t) a^{T} & p_{g \infty}(t) b_{g}^{T} \\
\hline 0 & 0
\end{array}\right)
$$




\section{B. NAGEL, Solutions of coupled diffusion equations}

(90) gives the asymptotic distribution $p_{\infty}(t)$ from the distribution $p_{0}$ at $t=0$ through

$$
p_{\infty}(t)=P^{\infty}(t) p_{0}
$$

(90) and (91) can also be rewritten in the following way. We express the initial distribution in terms of the component vectors belonging to the groups of essential states and the group of unessential states:

$$
p_{0}=\left\{\alpha_{1} p_{01}, \ldots \alpha_{g} p_{0 g}, \alpha_{R} p_{0 R}\right\},
$$

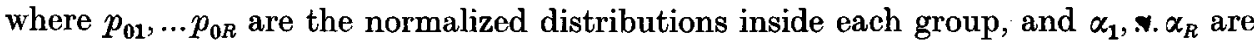
non-negative numbers, whose sum is one.

Then we can write the asymptotic distribution in the form

$$
p_{\infty}(t)=\left\{\left(\alpha_{1}+\alpha_{R} b_{1}^{T} p_{0 R}\right) p_{1 \infty}(t), \ldots\left(a_{g}+\alpha_{R} b_{g}^{T} p_{0 R}\right) p_{g \infty}(t), 0\right\}
$$

Finally we make some comments on the following problem, related to the question whether $\exp R_{i} t$ in (73) can be chosen to be a stochastic matrix for arbitrary $t$ even for non-constant $Q(t)$ :

What are the conditions on a stochastic matrix $P$ in order that it can be taken as the transition matrix (over a time interval 1, say) of a homogeneous Markov chain in continuous $t$, i.e. can be put in the form

$$
P=\exp Q
$$

where $Q$ is real, has non-negative elements outside the diagonal and fulfills $a^{T} Q=0$ ?

Evidently $P$ must have a positive determinant and positive diagonal elements. For $n=2$ these conditions are also sufficient. Namely, if

$$
Q=\left(\begin{array}{rr}
-a & b \\
a & -b
\end{array}\right), a, b \text { real, non-negative }
$$

we find

$$
\exp Q=\left(\begin{array}{c}
1-\frac{a}{a+b}\left(1-e^{-a-b}\right) \frac{b}{a+b}\left(1-e^{-a-b}\right) \\
\frac{a}{a+b}\left(1-e^{-a-b}\right) 1-\frac{b}{a+b}\left(1-e^{-a-b}\right)
\end{array}\right),
$$

and it is not difficult to verify that every stochastic $2 \times 2$ matrix with positive diagonal elements and positive determinant can be represented in this way for some non-negative $a, b$.

But for $n>2$ these conditions are not sufficient. From (94) follows, with $m$ chosen so that $Q+m E$ has positive diagonal elements, that

$$
P=e^{-m}\left[E+(Q+m E)+\frac{1}{2}(Q+m E)^{2}+\cdots\right]
$$

This relation implies that in the normal form of $P$ corresponding to (69) (where we thus also, if necessary, subdivide $P_{R}$ in (67)) all submatrices in the diagonal must be positive. In particular, if $P$ is positively regular, it must be positive. But for 
$n>2$ it is easy to find a positively regular stochastic matrix with positive determinant and positive diagonal and having an element equal to zero. Take $n=3$, $p_{21}=0$, and all other elements $>0$, with the diagonal elements near to 1 to ensure $|P|>0$. Such a matrix can also be taken to be generated by an inhomogeneous Markov chain in continuous time by choosing

$$
Q(t)=\left(\begin{array}{ccc}
-b(t) & x & x \\
0 & x & a(t) \\
b(t) & x & x
\end{array}\right)
$$

where

$$
\begin{aligned}
& a(t)>0 \text { for } 0<t<T ; \quad b(t)>0 \text { for } t>T ; \\
& a(t) b(t)=0, \quad \text { all } t \geqslant 0 \quad \text { (these relations imply } \\
& \left.a(t) b\left(t^{\prime}\right)=0, \quad t \geqslant t^{\prime} \geqslant 0\right) . \\
& x \text { denote non-zero elements. }
\end{aligned}
$$

$P(t)$ is then evidently positively regular for $t>T$, but a closer investigation of the series expression for $P(t)$ obtained from (2l) shows that $p_{21}(t)=0$, all $t \geqslant 0$.

\section{Asymptotic hehaviour of the Markovian process for large $t$ in the time-periodic case}

We shall now derive the asymptotic behaviour of $G(x, t)$ for large $t$ in the case where $Q(t), A(t)$, and $B(t)$ are periodic with period 1. As for the ordinary diffusion equation we expect that the probability diffuses towards infinity in $x$-space. This means that we should study $P(t, p)$ for small $p$ as $t \rightarrow \infty$.

As $A(t)$ and $B(t)$ are diagonal matrices, $P(t, p)$ decomposes in the same way as $P(t)$, and we get equations analogous to $(70)-(72)$. We first study the equation corresponding to $(70)$, describing the behaviour inside a group of essential states. For simplicity we omit the index $i$ :

$$
\frac{\partial}{\partial t} P(t, p)=\left[Q(t)+i p A(t)-\frac{1}{2} p^{2} B(t)\right] P(t, p), \quad P(0, p)=E .
$$

As for (70) we can write the solution in the form

$$
P(t, p)=S(t, p) \exp [R(p) t],
$$

where $S(t, p)$ is periodic and $S(0, p)=E$.

We denote by $\lambda(p)$ the eigenvalue of $P(1, p)$ which takes the value one for $p=0$, and by $\lambda_{i}(p)$ the remaining eigenvalues. From continuity follows that $\lambda(p)$ is welldefined and a simple eigenvalue for $p$ small enough, as $\lambda(0)=1$ is a simple eigenvalue. As an algebraic function of the matrix elements of $P(1, p)$, which are integral functions of $p, \lambda(p)$ is even an analytic function in a neighbourhood of $p=0$ (cf. e.g. [8], Ch. 5 , p. 125; this reference studies the case where the coefficients of the characteristic equation determining $\lambda(p)$ are polynomials in $p$, but the proof applies also to the case where the coefficients are analytic functions in a neighbourhood of $p=0$ ). Then 


\section{B. NAGEL, Solutions of coupled diffusion equations}

also $\mu(p)=\ln \lambda(p)$ (principal value) is analytic in a neighbourhood of $p=0$, and we have the expansion

$$
\ln \lambda(p)=\mu(p)=\mu^{\prime} p+\frac{1}{2} \mu^{\prime \prime} p^{2}+\cdots
$$

convergent for $p$ small enough.

In analogy with (75) we get from (74), using $A=P(1, p)$ and $f(\lambda)=\lambda^{t}$

$$
\exp [R(p) t]-\exp [\mu(p) t] \cdot P^{\infty}(p)=0\left(e^{-\alpha t}\right), \text { small } p .
$$

If one looks a little closer at the way the polynomials $Z_{k l}$ of $A$ in (74) are defined (see [5]), it is seen that the matrix elements of $Z_{k l}$ are bounded if $p$ varies in a bounded region of the $p$-plane. Since $\left|\lambda_{i}(0)\right|<1$, we can always find a $\delta>0$ such that

$$
\operatorname{Re} \mu(p)>\operatorname{Re} \ln \lambda_{i}(p) \text { for }|p| \leqslant \delta .
$$

Then the expression

$$
\exp [-\mu(p) t] \exp [R(p) t]-P^{\infty}(p)
$$

converges to zero as $t \rightarrow \infty$, uniformly in $p$ for $|p| \leqslant \delta$.

$\operatorname{Exp}[R(p) N]=[P(1, p)]^{N}, N$ positive integer, is an integral function of $p . P^{\infty}(p)$ must then be analytic for $|p| \leqslant \delta$, because (105) shows that it is the uniform limit of a sequence of analytic functions. It is also well known from the theory of functions that in this case also all derivatives of the sequence of analytic functions converge uniformly to the corresponding derivatives of the limit function. Applied to (103), with $t=N$, this shows that all derivatives of the left hand side with respect to $p$ converge to 0 as $t \rightarrow \infty$, for $p$ small enough. This fact will be used later on when we derive expressions for $\mu^{\prime}$ and $\mu^{\prime \prime}$.

If we put $p=i q$, purely imaginary, in $(100)$, it is seen that $P(t, i q)$ is real. Then $\lambda(i q)$ must stay real for small real $q$, as non-real eigenvalues of real matrices occur in complex conjugate pairs, and $\lambda(0)$ is a simple eigenvalue. This means that in the expansion (102) $\mu^{\prime}$ must be imaginary (or zero), and $\mu^{\prime \prime}$ real. We found in the beginning of Section III that the eigenvalues of $P(t, p), p$ real, are bounded by 1 in modulus. Then $\mu^{\prime \prime}$ must be non-positive. We thus put

$$
\mu^{\prime}=i m, \quad \mu^{\prime \prime}=-2 D .
$$

Expressions for $m$ and $D$ in terms of $P(t), A(t)$, and $B(t)$ will be derived below. We shall assume

$$
D>0
$$

this is true, e.g. if $B(t)$ is not identically zero.

We put

$$
\begin{gathered}
p_{1}=p \sqrt{2 D t}, \\
t=N+\tau, \quad 0 \leqslant \tau<1, \quad \tau \text { fixed, } \quad N \text { integer, }
\end{gathered}
$$

and study the limit as $N \rightarrow \infty$ of 


$$
\bar{P}_{N}\left(\tau, p_{1}\right)=e^{-i m p t} P(t, p)=\exp \left[-\frac{1}{2} p_{1}^{2}+0(1 / \sqrt{t})\right] S\left(\tau, p_{1} / \sqrt{2 D t}\right) P^{\infty}\left(p_{1} / \sqrt{2 D t}\right)+0\left(e^{-\alpha t}\right)
$$

which is obtained from (101) and (103).

Then for any $p_{1}$

$$
\lim _{N \rightarrow \infty} \bar{P}_{N}\left(\tau, p_{1}\right)=\exp \left(-\frac{1}{2} p_{1}^{2}\right) p_{\infty}(\tau) a^{T}
$$

where we have used (78) and (80). The convergence is even uniform in every bounded set in the $p_{1}$-plane, especially in any finite interval of the real $p_{1}$-axis.

The probability density corresponding to $\bar{P}_{N}\left(\tau, p_{1}\right)$,

$$
\bar{G}_{N}\left(x_{1}, \tau\right)=\frac{1}{2 \pi} \int \bar{P}_{N}\left(\tau, p_{1}\right) \exp \left(-i p_{1} x_{1}\right) d p_{1},
$$

where

$$
x_{1}=(x-m t) / \sqrt{2 D t}
$$

is related to $G(x, t)$ by $G(x, t)=\bar{G}_{N}\left(\frac{x-m t}{\sqrt{2 D t}}, \tau\right) / \sqrt{2 D t}$.

We now assume that the quantity $b$ defined in (54) (cf. Theorem 4) is positive. As $B(t)$ is periodic, $b$ increases asymptotically proportional to $t$, and introducing $p_{1}$ from (108) into $(56)$ we find that $\bar{P}_{N}\left(\tau, p_{1}\right)$ are bounded uniformly in $N$ on the entire real $p_{1}$-axis by some Gaussian:

$$
\left|\left\{\bar{P}_{N}\left(\tau, p_{1}\right)\right\}_{i k}\right|<A \exp \left(-\alpha p_{1}^{2}\right), \quad A \text { and } \alpha>0 \text { independent of } N .
$$

Dividing the region of integration in (112) into two parts, $\left|p_{1}\right|<L$ and $\left|p_{1}\right| \geqslant L$, respectively, and using (111) (which holds uniformly in $\left|p_{1}\right|<L$ ) and (115) (which shows that the contribution from $\left|p_{1}\right| \geqslant L$ is negligible, uniformly in $N$, for $L$ large enough), combined with

$$
\frac{1}{2 \pi} \int \exp \left(-\frac{1}{2} p_{1}^{2}-i p_{1} x_{1}\right) d p_{1}=\frac{1}{\sqrt{2 \pi}} \exp \left(-\frac{1}{2} x_{1}^{2}\right),
$$

we find

$$
\lim _{N \rightarrow \infty} \bar{G}_{N}\left(x_{1}, \tau\right)=\frac{1}{\sqrt{2 \pi}} \exp \left(-\frac{1}{2} x_{1}^{2}\right) p_{\infty}(\tau) a^{T}
$$

where the convergence is uniform in $x_{1}$. As all $\bar{G}_{N}\left(x_{2}, \tau\right)$ are integral functions of $x_{1}$ (cf. Sect. II), this uniform convergence implies the uniform convergence of all derivatives to the derivatives of the right hand side of (117).

Introducing $G(x, t)$ with the help of (114), (117) can be written in a somewhat unprecise way as

$$
G(x, t) \sim \frac{1}{\sqrt{4 \pi D t}} \exp \left(-\frac{(x-m t)^{2}}{4 D t}\right) p_{\infty}(\tau) a^{T}, \quad \text { large } t .
$$




\section{B. NAGEL, Solutions of coupled diffusion equations}

In a similar way the generalization of (72) to the case $p \neq 0$ leads to

or

$$
\begin{gathered}
\lim _{N \rightarrow \infty} \bar{P}_{i R_{N}}\left(\tau, p_{1}\right)=\exp \left(-\frac{1}{2} p_{1}^{2}\right) p_{i \infty}(\tau) b_{i}^{T}, \\
G_{i R}(x, t) \sim \frac{1}{\sqrt{4 \pi D t}} \exp \left(-\frac{(x-m t)^{2}}{4 D t}\right) p_{i \infty}(\tau) b_{i}^{T}, \quad \text { large } t .
\end{gathered}
$$

Here we have kept the index $i$ to avoid confusion with $\boldsymbol{r}_{R}(t, p)$ etc. Hence $p_{\infty}(\tau)$ in (118) and $p_{i \infty}(\tau)$ in (119) are the same.

(118) and (120) mean that inside each group of essential states the distribution in $x$-space is asymptotically the same for all states and normal with mean value $m t$ and variance $2 D t$.

The assumption $b>0$ enabled us to deduce the strong convergence (117) to the normal distribution, using elementary methods. If we drop this assumption, (117) is still valid in a weaker sense, as follows from a fundamental theorem for sequences of characteristic functions (see e.g. [4] Ch. VII):

If a sequence of characteristic functions $\left\{f_{v}(p)\right\}$ converges for all real $p$ to a continuous $f(p)$ (which is then also a characteristic function), then the corresponding distribution functions $\left\{F_{\nu}(x)\right\}$ converge weakly to $F(x)$, the distribution function corresponding to $f(p)$. Weak convergence can be taken to mean that $F_{v}(x) \rightarrow F(x)$ in all continuity points of $F(x)$.

Applied to our case this theorem implies that (117) is true if we take the limit after integrating both sides over some fixed interval in $x_{1}$. (118) and (120) should be interpreted correspondingly.

What we have found concerning the asymptotic convergence (in a strong or weak sense) of $G(x, t)$ to a normal distribution carries over directly to the distribution $p(x, t)$ corresponding to an arbitrary initial distribution $p(x, 0)$, as one easily sees from (37). No assumptions concerning the existence of moments of order $\geqslant 1$ for $p(x, 0)$ are necessary.

It remains to derive explicit expressions for $m$ and $D$. We start from (103) for $t=N$, differentiate two times with respect to $p$, and put $p=0$. This gives

$$
\begin{gathered}
(\partial P / \partial p)(N, 0)=N \mu^{\prime} P^{\infty}+\left(\partial P^{\infty} / \partial p\right)(0)+\varepsilon \\
\left(\partial^{2} P / \partial p^{2}\right)(N, 0)=N \mu^{\prime \prime} P^{\infty}+N^{2} \mu^{\prime 2} P^{\infty}+2 N \mu^{\prime}\left(\partial P^{\infty} / \partial p\right)(0)+0(1) .
\end{gathered}
$$

: and $0(1)$ denote quantities which vanish, or stay bounded, respectively, as $N \rightarrow \infty$.

$$
a^{T} P^{\infty} p_{\infty}=1, \quad \text { (cf. (78)) }
$$

we find

$$
\begin{gathered}
\mu^{\prime}=\lim _{N \rightarrow \infty} \frac{1}{N} a^{T}(\partial P / \partial p)(N, 0) p_{\infty} \\
\mu^{\prime \prime}=\lim _{N \rightarrow \infty} \frac{1}{N}\left[a^{T}\left(\partial^{2} P / \partial p^{2}\right)(N, 0) p_{\infty}-\left\{a^{T}(\partial P / \partial p)(N, 0) p_{\infty}\right\}^{2}\right] .
\end{gathered}
$$

Equations for the derivatives of $P$ occurring in (123) and (124) are obtained from (100) by differentiating both sides with respect to $p$, cf. the remark at the beginning of Sect. II: 


$$
\begin{gathered}
\frac{\partial}{\partial t}\left[a^{T}(\partial P / \partial p)(t, 0) p_{\infty}\right]=i a^{T} A(t) S(t) p_{\infty} \\
\frac{\partial}{\partial t}\left[a^{T}\left(\partial^{2} P / \partial p^{2}\right)(t, 0) p_{\infty}\right]=2 i a^{T} A(t)(\partial P / \partial p)(t, 0) p_{\infty}-a^{T} B(t) S(t) p_{\infty} .
\end{gathered}
$$

Here we have used

$$
P(t) p_{\infty}=S(t) e^{R t} p_{\infty}=S(t) p_{\infty}=p_{\infty}(t)
$$

cf. eq. (83), and

$$
a^{T} Q(t)=0 .
$$

Furthermore, as $a^{T}(\partial P / \partial p)(t, 0) p_{\infty}$ is a number, (125) shows that

$$
\frac{\partial}{\partial t}\left[a^{T}(\partial P / \partial p)(t, 0) p_{\infty}\right]^{2}=2 i a^{T} A(t) S(t) p_{\infty} a^{T}(\partial P / \partial p)(t, 0) p_{\infty}
$$

As $p_{\infty} a^{T}=P^{\infty},(126)$ and (129) give

$$
\begin{aligned}
& \frac{\partial}{\partial t}\left[a^{T}\left(\partial^{2} P / \partial p^{2}\right)(t, 0) p_{\infty}-\left\{a^{T}(\partial P / \partial p)(t, 0) p_{\infty}\right\}^{2}\right] \\
& \quad=-a^{T} B(t) S(t) p_{\infty}-2 i a^{T} A(t)\left[S(t) P^{\infty}-E\right](\partial P / \partial p)(t, 0) p_{\infty}
\end{aligned}
$$

For $(\partial P / \partial p)(t, 0) p_{\infty}$ we have the equation

$$
\frac{\partial}{\partial t}\left[(\partial P / \partial p)(t, 0) p_{\infty}\right]=Q(t)\left(\partial P / \partial p(t, 0) p_{\infty}+i A(t) P(t) p_{\infty}\right.
$$

which is solved in the same way as (72), see (84)-(86):

$$
(\partial P / \partial p)(t, 0) p_{\infty}=i P(t) \int_{0}^{t} e^{-R \tau} S^{-1}(\tau) A(\tau) S(\tau) p_{\infty} d \tau .
$$

Introducing (132) into (130), integrating (125) and (130) from 0 to $N$, and observing that

$$
P^{\infty} P(t)=p_{\infty} a^{T} P(t)=P^{\infty}
$$

we find the following expressions for $m$ and $D$ :

$$
\begin{gathered}
m=\int_{0}^{1} a^{T} A(t) p_{\infty}(t) d t \\
2 D=\int_{0}^{1} a^{T} B(t) p_{\infty}(t) d t+2 \lim _{N \rightarrow \infty} \frac{1}{N} \int_{0}^{N} d t a^{T} A(t) S(t) \\
\times \int_{0}^{t} d \tau\left[e^{R(t-\tau)}-P^{\infty}\right] S^{-1}(\tau) A(\tau) p_{\infty}(\tau) .
\end{gathered}
$$




\section{B. NAGEL, Solutions of coupled diffusion equations}

In (134) and the first term of (135) we have used the fact that $A(t), B(t)$, and $p_{\infty}(t)$ are periodic. These terms are the mean values of the diagonal elements of $A(t)$ and $B(t)$ over a period of the asymptotic distribution $p_{\infty}(t)$. The second term in (135), which is the term we called the electrodiffusion component in Sect. $I$, is non-negative, because even if we put $B(t)=0, D$ is non-negative, as we have shown.

If $A(t)=a(t) E$, i.e. $A(t)$ has all diagonal elements equal, we get in (130)

$$
a^{T} A(t)\left[S(t) P^{\infty}-E\right]=a(t)\left[a^{T} S(t) P^{\infty}-a^{T}\right]=0,
$$

i.e. the second term in (135) gives no contribution to $D$ in this case.

We shall also give the somewhat simplified forms of $m$ and $D$ that are obtained if $Q(t)=Q$ is constant, and hence $S(t)=E, R=Q . A(t)$ and $B(t)$ have the Fourier expansions

$$
\begin{aligned}
& A(t) \sim \sum_{-\infty}^{\infty} A_{\nu} e^{i 2 \pi v t}, \\
& B(t) \sim \sum_{-\infty}^{\infty} B_{\nu} e^{i 2 \pi v t},
\end{aligned}
$$

and for $e^{Q t}$ we can write, according to (74),

$$
e^{Q t}=P^{\infty}+\sum_{k=1}^{s} \sum_{\mu=0}^{m_{k}-1} t^{\mu} e^{-\alpha_{k} t} Z_{k, \mu+1}
$$

Here $\alpha_{k}$, which are equal to minus the non-zero eigenvalues of $Q$, have positive real parts.

Using (137) and (138) in (134) and (135) we get after some straightforward calculations

$$
\begin{gathered}
m=a^{T} A_{0} p_{\infty} \\
2 D=a^{T} B_{0} p_{\infty}+2 \sum_{\nu=-\infty}^{\infty} \sum_{k, \mu} a^{T}\left(A_{-\nu} Z_{k \mu+1} A_{\nu}\right) p_{\infty} \frac{\mid \mu}{\left(\alpha_{k}+i 2 \pi v\right)^{\mu+1}} .
\end{gathered}
$$

\section{Application to electrodiffusion. Generalizations}

We now apply our general theory to the electrodiffusion equations (1), where

$$
Q=\left(\begin{array}{cc}
-k_{1} & k_{2} \\
k_{1} & -k_{2}
\end{array}\right), \quad A(t)=g(t)\left(\begin{array}{ll}
u_{1} & 0 \\
0 & u_{2}
\end{array}\right), \quad B(t)=2\left(\begin{array}{ll}
D_{1} & 0 \\
0 & D_{2}
\end{array}\right) .
$$

We assume $k_{1}$ and $k_{2}$ positive, and put

$$
k=k_{1}+k_{2} \text {. }
$$

As $Q(t)$ is constant, $P(t)$ is easy to compute, ef. (96):

$$
P(t)=e^{Q t}=\frac{1}{k}\left(\begin{array}{ll}
k_{2}+k_{1} e^{-k t} & k_{2}\left(1-e^{-k t}\right) \\
k_{1}\left(1-e^{-k t}\right) & k_{1}+k_{2} e^{-k t}
\end{array}\right)
$$


which gives

$$
P^{\infty}=\frac{1}{k}\left(\begin{array}{ll}
k_{2} & k_{2} \\
k_{1} & k_{1}
\end{array}\right), \quad p_{\infty}=\left\{k_{2} / k, k_{1} / k\right\}
$$

Given initial normalized concentrations $\left\{c_{i}(x, 0)\right\}$, we saw in Sect. II that $\left\{c_{i}(x, t)\right\}$ are for $t>0$ integral functions of $x$, provided $D_{1}$ and $D_{2}$ are positive. $\left\{c_{1}(x, t)\right\}$ have moments up to the same order as $\left\{c_{i}(x, 0)\right\}$.

We now assume $g(t)$ periodic, period $2 \pi / \omega$, with the Fourier expansion

$$
g(t) \sim \sum_{-\infty}^{\infty} g_{\nu} e^{i \omega v t}, \quad g_{-\nu}=g_{v}^{*}
$$

As we assumed, for simplicity, a period 1 in Sect. IV, we must normalize the time variable in (1), putting $t^{\prime}=\omega t / 2 \pi$; before we apply the formulas derived in Sect. IV.

Asymptotically we have the behaviour

$$
\left\{c_{1}(x, t), c_{2}(x, t)\right\} \sim \frac{1}{\sqrt{4 \pi D t}} \exp \left(-\frac{(x-m t)^{2}}{4 D t}\right)\left\{k_{2} / k, k_{1} / k\right\}
$$

where

$$
\begin{gathered}
m=\frac{1}{k}\left(u_{1} k_{2}+u_{2} k_{1}\right) g_{0} \\
D=\frac{1}{k}\left(D_{1} k_{2}+D_{2} k_{1}\right)+\left(u_{1}-u_{2}\right)^{2} \frac{k_{1} k_{2}}{k^{3}}\left[g_{0}^{2}+2 \sum_{v=1}^{\infty} \frac{\left|g_{v}\right|^{2}}{1+(\omega \nu)^{2} / k^{2}}\right] .
\end{gathered}
$$

The results (146)-(148) are equivalent to those obtained by Thedéen, using a twostate random walk model [3]. It should be remarked that our continuity requirement on the function $g(t)$ is stronger than the condition of integrability assumed by Thedéen.

Finally we would like to mention some possible generalizations of the problem. studied in this paper.

The generalization to the case where the $x$-space is $m$-dimensional, $x=\left(x_{1}, \ldots x_{m}\right)$, is almost trivial, Instead of (8) we obtain

$$
\frac{\partial}{\partial t} G(x, t)=\left[Q(t)-A^{i}(t) \frac{\partial}{\partial x_{i}}+\frac{1}{2} B^{i k}(t) \frac{\partial^{2}}{\partial x_{i} \partial x_{k}}\right] G(x, t),
$$

(summation from 1 to $m$ over repeated indices) where e.g., cf. (R 4),

$$
B^{i k}(\tau)=\lim \frac{1}{\Delta \tau} \int x_{i} x_{k} Q(x, \tau+\Delta \tau ; 0, \tau) d x_{1} \ldots, d x_{m} .
$$

All $A^{i}(t)$ and $B^{i k}(t)$ are diagonal matrices. Positivity (or non-negativity) of the diagonal elements of $B(t)$ is generalized to positive definiteness (or positive semidefiniteness) of the diagonal elements of $p_{i} p_{k} B^{i k}(t)$ as quadratic forms in $p_{i}$. The analysis in Sect. II is modified in an obvious way, whereas III is of course unchanged. Corresponding to the results of IV we find inside each group of essential states an 


\section{B. NAGEL, Solutions of coupled diffusion equations}

asymptotic approach to a $m$-dimensional normal distribution, determined by $m$ first order moments $m_{i} t$ and $\frac{1}{2} m(m+1)$ second order central moments $2 D_{i k} t\left(D_{i k}=D_{k i}\right)$. $m_{i}$ and $D_{i k}$ are determined by formulas obtained from (134) and (135) by proper indexing of $m, D, A(t)$, and $B(t)$ in these formulas. In the last term in (135) we have to form half the sum of the expressions with $A_{i}(t) \ldots A_{k}(\tau)$ and $A_{k}(t) \ldots A_{i}(\tau)$.

Probably less trivial is the generalization to the case $n=\infty$, i.e. when we have a countably infinite number of discrete states. We shall not consider this case here.

\section{A CKNOWLEDGEMENTS}

It is a pleasure to thank Dr. S. Ljunggren and Dr. T. Thedéen for informative discussions on the problem of electrodiffusion.

Division of Mathematical Physics, Royal Institute of Technology, Stockholm 70, Sweden

\section{R E F E R E N C E S}

1. BaK, T. A., and KaUman, W. G., Theory of electrodiffusion. Trans. Faraday Soc. 55, 1109 (1959).

2. L.JUNGGREN, S., A study of electrodiffusion and of diffusion combined with kineties by means of moment analysis. Trans. Roy. Inst. Tech., Stockholm. No. 142 (1959).

3. Thedéen, T., Electrodiffusion treated as a random walk problem. Trans. Roy. Inst. Tech., Stockholm. No. 165 (1960).

4. Gredenko, B. V., Lehrbuch der Wahrscheinlichkeitsrechnung (German transl. of Russian original, Akademie-Verlag, Berlin 1958); The Theory of Probability (English transl., Chelsea 1962).

5. Gantmacher, F. R., Matrizenrechnung I, II (German transl. of Russian original, DVW, Berlin 1958-59); The Theory of Matrices I, II (English transl., Chelsea 1959).

6. Bartiett, M. S., Stochastic Processes (Cambridge 1955).

7. Codprngton, E. A., and Levinson, N., Theory of Ordinary Differential Equations (McGrawHill 1955).

8. KNopp, K., Theory of Functions II (English transl. of German original, Dover 1947). 